\section{Alternative Perspectives on Students' Reasoning about Emergent Processes}

\section{Julia Gouvea*}

Tufts University, Medford, MA 02155

M any patterns in the biological world-diffusion, flocking, natural selection, and human cognition-appear as though driven by some intentional agent or central organizing force. Experts understand these processes as emerging from collective interactions among individual agents. For example, microlevel interactions among colliding molecules cause diffusion, not directed flow. Understanding the distinction between directed and emergent processes is considered central to learning to think biologically, but teaching students about these processes is a challenge, even at the undergraduate level (e.g., Garvin-Doxas and Klymkowsky, 2008).

In this installment of Current Insights, I highlight two recent and different perspectives on this problem. The first, represented by Chi et al. (2012), proposes that young learners generally lack the appropriate cognitive structures to correctly identify and explain emergent processes and therefore need to be taught how to think about emergence through direct instruction. The second, represented by Grotzer et al. (2017), argues that young learners have prior experiences and knowledge (often referred to as "resources") related to emergent processes that can be built upon though instruction that features exploration and reflection. The contrast is useful not only because the two articles provide many insights on the possible nature of causal reasoning, but also because the authors are uncommonly articulate about their underlying theoretical assumptions. Reading the two together can provoke considerations of how alternative theories of learning can inform research and teaching.

Chi, M. T. H., Roscoe, R. D., Slotta, J. D., Roy, M., \& Chase, C. C. (2012). Misconceived causal explanations for emergent processes. Cognitive Science, 36(1), 1-61. https://doi.org/10.1111/j.1551-6709.2011.01207.x

Chi and colleagues begin their article by outlining their stance on the development of emergent reasoning. Their core assumption is that children think about natural processes as sequential and directed. This assumption is based in part on related literature on how children understand stories. Stories tend to have a beginning, a series of linked events, and a defined ending. They also tend to have central characters whose actions drive the narrative forward. Chi and colleagues propose that early childhood experiences with the world often have this linear sequential form, leading children to develop generalized "direct-causal schemas" that they apply broadly. When applied to emergent processes, the authors argue, a direct-causal schema leads to misconceptions.

Chi and colleagues further assume that children's experiences in the world do not lead them to develop an appropriate "emergent schema" that would include ideas about agent-level interactions. The authors suggest that ideas about emergence need to be taught directly, because children lack this schema.

Next, the authors describe an instructional intervention in the form of an online module that combines explicit instruction about the general structure of emergent processes (e.g., that underlying agent behavior is uniform and random), readings that connect general ideas to the specific case of diffusion, and guided interactions with a computer simulation representing diffusion of dye in water at the macroand microlevels.
CBE Life Sci Educ March 1, 2018 17:fe2 DOI:10.1187/cbe.18-01-0001

This feature is designed to point CBE-Life Sciences Education readers to current or noteworthy articles for life science educators and education researchers. We invite readers to suggest current themes or articles of interest in life science education as well as influential papers published in the more distant past or in the broader field of education research to be featured in Current Insights. Please send any suggestions to Julia.Gouvea@itufts.edu.

*Address correspondence to: Julia Gouvea (Julia.Gouvea@tufts.edu).

(C) 2018 J. Gouvea. CBE-Life Sciences Education (C) 2018 The American Society for Cell Biology. This article is distributed by The American Society for Cell Biology under license from the author(s). It is available to the public under an Attribution-Noncommercial-Share Alike 3.0 Unported Creative Commons License (http:// creativecommons.org/licenses/by-nc-sa/3.0). "ASCB ${ }^{\circledR "}$ and "The American Society for Cell Biology ${ }^{\circledR "}$ are registered trademarks of The American Society for Cell Biology. 
The researchers tested this emergence-oriented intervention with eighth- and ninth-grade students and compared this group's performance on a variety of assessments with that of a group of students who received a module that included "filler" text on the nature of science in the place of emergent processes. Both groups read about diffusion and interacted with the simulation, but the emergence group received specific prompts related to noticing emergent properties of diffusion, while the comparison group did not. The emergent process group made larger gains on a diffusion posttest than the comparison group. Further, the gains were larger for those students within the emergence group who demonstrated a stronger general understanding of emergent processes.

Chi and colleagues acknowledge that the success of their intervention is not conclusive evidence of their proposed cognitive hypothesis, but conclude that its success supports the idea that teaching students about emergent processes in general could help them overcome a wide range of specific misconceptions.

Grotzer, T. A., Derbiszewska, K., \& Solis, S. L. (2017). Leveraging fourth and sixth graders' experiences to reveal understanding of the forms and features of distributed causality. Cognition and Instruction, 35(1), 55-87. https://doi.org/ 10.1080/07370008.2016.1251808

Grotzer and colleagues approach this problem from a different set of starting assumptions. The authors start from the assumption that children have prior experiences that they can use to make sense of emergent phenomena. They imagine, for example, that children could experience the rising volume in a cafeteria at lunchtime and understand it to arise from individual conversations getting louder and louder. To identify these potential resources, the authors conducted interviews with students before and after an instructional intervention.

The intervention was designed specifically to connect to students' everyday experiences with emergent processes, including those that researchers observed in the school (like the cafeteria example). In addition, it included activities that built up experiences by having students participate in embodied simulations of emergent and directed processes (lining up or moving randomly) and viewing video examples of both directed and emergent natural phenomena. Opportunities for discussion and reflection were supported throughout.

Through qualitative coding of interviews conducted before and after the intervention, the authors analyzed both the nature and frequency of different patterns of reasoning in fourth- and sixth-grade students. They found evidence of various forms of decentralized and emergent reasoning in students' pre interviews (in $73 \%$ of instances) as well as gains in post interviews (78\% of instances).

What is perhaps more interesting is that Grotzer and colleagues provide in-depth descriptions of the various forms of students' reasoning that emerged from their analysis. To provide just one example, the authors describe that students did not strictly categorize processes as either directed or emergent; rather, they were flexible in their characterizations, attending to relevant features of context. For example, when asked to explain the pattern of children sitting evenly spaced on a rug, one student offered up two alternative explanations: it could be because the teacher directed children to sit in assigned places or it could be the result of each child independently choosing a place to sit. The authors describe other examples of students providing flexible or hybrid explanations that demonstrate an appreciation of the nuances of specific situations rather than application of a generalized schema.

Overall, Grotzer and colleagues argue that building on students' everyday experiences through reflection and exploration can be an effective way to expand upon existing ideas children may have about emergent processes. The authors acknowledge that the study is limited, particularly in terms of sample size. Still, they argue that their study provides evidence of young students having more ideas and experiences relevant to emergent processes than predicted by prior research (e.g., Chi et al., 2012).

While these two papers differ in their theoretical orientations (a missing schema vs. resources from prior experiences) and instructional implications (direct instruction vs. reflection and discussion of everyday phenomena), they both build the case that students in the middle and elementary grades have the capacity to learn about emergent processes. Insights from these two studies of younger learners may therefore inform what we can expect of college-age students, and comparing and contrasting the two approaches may inspire ideas for research and instructional design at the undergraduate level.

URLs are provided for the abstracts or full text of articles.

\section{REFERENCE}

Garvin-Doxas, K., \& Klymkowsky, M. W. (2008). Understanding randomness and its impact on student learning: Lessons learned from building the Biology Concept Inventory (BCI). CBE-Life Sciences Education, 7(2), 227-233. https://doi.org/10.1187/cbe.07-08-0063 